\title{
ERRATA, VOLUME 85
}

The Fatou theorem and its converse. By F. W. Gehring. Pages 106-121.

Page 107, Lemma 1. For " $k(x)$ is in $M f(x)$ " read " $k(x)$ is in $M$ if $f(x) . "$ Page 112, Theorem 3. For " $D_{\downarrow} u\left(r e^{i \theta}\right)=O^{n}\left(r^{\delta-n}\right)$ " read " $D_{\downarrow}^{n} u\left(r e^{i \theta}\right)=O\left(r^{\delta-n}\right) . "$ Page 114, Theorem 5. For " $A y\left(x^{2}+y^{2}\right)$ " read " $A y /\left(x^{2}+y^{2}\right)$."

Page 116, Theorem 9. For " $D_{d}^{n}\left(r e^{i b}\right)$ " read " $D_{d}^{n} u\left(r e^{i b}\right)$."

Page 116, Theorem 10. For " $(b-a) \delta+R-\mu \neq 0$ " read “ $(b-a) \delta+\eta-\mu \neq 0$." Page 116, Theorem 10. For " $B r^{\delta-m}$ " read " $B r^{\delta-n "}$. 